\title{
High-energy Emission from Supernovae and Remnants
}

\author{
R. A. Chevalier \\ Department of Astronomy, University of Virginia, P.O. Box 3818, \\ Charlottesville, VA 22903, U.S.A.
}

\begin{abstract}
.
An early burst of energetic radiation is expected from a supernova at the time of shock breakout. This emission has not been directly observed but has been inferred from the photoionization around SN 1987A. X-ray emission has been detected from core-collapse supernovae in the days to years after the explosion as they interact with their circumstellar winds. Young Galactic supernova remnants provide the possibility of determining the composition structure of the ejecta through $\mathrm{X}$-ray spectroscopy. An exciting finding for older remnants is that a number of remnants that appear to be interacting with molecular gas may be sources of high-energy $\gamma$-ray emission. The clumpy structure of molecular clouds has implications for the structure expected in high-energy emission. Finally, the field of $\gamma$-ray-line spectroscopy is beginning to yield results relevant to the explosive nucleosynthesis of radionuclides in supernovae.
\end{abstract}

\section{Introduction}

The study of high-energy emission from supernova remnants has a long history because remnants are among the brightest sources in the sky. The Crab Nebula has long been a target of high-energy experiments and opened up the study of high-energy, nonthermal emission. The emission is related to the power deposition of the central pulsar into a relativistic wind. Another bright supernova remnant is Cas A, which is characterized by thermal emission from shock-heated gas.

The Crab Nebula and Cas A provide examples of two of the power sources for high-energy emission from supernovae and their remnants: pulsars and the explosion shock wave. A third power source, which has been directly observed only more recently, is radioactive decay. In the present review, I do not treat the pulsar-related emission because it is covered by Becker (2000) and Pacini (2000) in these Proceedings (see also Chevalier 1998). In Section 2, the shock-related emission is discussed, beginning with the initial shock breakout from the stellar photosphere. The shock wave then moves into circumstellar material. X-rays have now been detected from a number of extragalactic supernovae. The topic of thermal emission from galactic SNRs is a large one, and I give only some examples. Nonthermal X-ray emission from remnants is treated more fully by Petre (2000, these Proceedings) but is mentioned here. Finally, older remnants moving into dense media can be sources of $\gamma$-ray emission. In Section 3 , high- 
energy emission related to radioactivity is briefly covered, and future prospects are discussed in Section 4.

\section{Shock Wave Interactions}

\subsection{Shock Breakout}

In a core-collapse supernova with a massive star progenitor, the central explosion generates a radiation-dominated shock wave that moves out through the star. In a red supergiant progenitor, the shock wave takes about one day to traverse the star. At shock breakout, the photospheric luminosity can be $\sim 10^{45} \mathrm{erg} \mathrm{s}^{-1}$ for 30 minutes with an effective temperature of $\sim 2 \times 10^{5} \mathrm{~K}$ (Klein \& Chevalier 1978; Falk 1978). The radiation temperature is higher than the effective temperature because of the scattering atmosphere. Matzner \& McKee (1999) have recently derived useful scaling relations that show how the properties of the prompt emission depend on the characteristics of the progenitor star.

The hard ultraviolet/soft X-ray burst from a supernova has not been observed, but we do have evidence for such a burst in SN 1987A. The early temperature estimates with IUE gave $T_{\text {eff }} \approx 15,000 \mathrm{~K}$ after a day (Kirshner et al. 1987), but models for the shock breakout imply peak temperatures of $\sim 3 \times 10^{5}$ $10^{6} \mathrm{~K}$ (e.g., Ensman \& Burrows 1992). Evidence for high temperatures comes from emission from the circumstellar gas heated and ionized by the flash. The early ultraviolet circumstellar lines, including NV, require a color temperature of $(1.0-1.5) \times 10^{6} \mathrm{~K}$ (Lundqvist \& Fransson 1996). Until recently, all the emission from the well-known rings around SN 1987A could be attributed to the initial heating by the radiation at shock breakout. Now, part of the ring is brightening as the forward shock front from the supernova appears to be interacting with a ring knot that is especially close to the supernova (Sonneborn et al. 1998).

The direct detection of the expected burst of hard ultraviolet/soft X-ray radiation would allow a determination of the stellar radius and would open the study of the very early evolution of supernovae. Harder radiation is expected from more compact progenitor stars. Type Ia supernovae with white dwarf progenitors should emit very energetic radiation at the time of shock breakout, but their small radius leads to a low luminosity and detection of such a burst is not promising. In any case, this expected, but as yet unobserved, phenomenon shows that the bursting sky probably contains interesting discoveries to be made.

\subsection{Supernovae}

At the time of shock breakout, the radiation-dominated shock wave becomes very broad and surrounding material is accelerated to a $v \propto r^{-2}$ velocity profile. The shock front disappears but reforms as a viscous shock front because of the decreasing velocity with radius (Murzina \& Chevalier, private comm.). The forward shock front initially has a velocity $\gtrsim 10^{4} \mathrm{~km} \mathrm{~s}^{-1}$ and can heat gas to $T \sim 10^{9} \mathrm{~K}$. Because of deceleration of the shocked gas, the supernova gas, which is freely expanding, can catch up with the shocked shell and form a reverse shock front. This gas has a higher density and a temperature $\sim 10^{7} \mathrm{~K}$.

$\mathrm{X}$-ray emission from a supernova has been best studied for the case of the Type II supernova SN 1993J in M81 (Fransson, Lundqvist, \& Chevalier 1996, 
and references therein). Initially, a very hard burst of radiation was observed with $T \sim 10^{9} \mathrm{~K}$. The emission properties indicate expansion into a wind with $\dot{M} \approx 4 \times 10^{-5} M_{\odot} \mathrm{yr}^{-1}$ for a wind velocity $v_{w}=10 \mathrm{~km} \mathrm{~s}^{-1}$, so the absorption by circumstellar gas could have been much more significant than was actually observed. The ionizing radiation from the supernova was capable of fully ionizing the circumstellar gas ahead of the forward shock front, reducing the X-ray absorption. The emission was initially observed to have no soft X-ray component, but, after 220 days, such a component was observed. A plausible reason for the absence of this component at early times is that the reverse shock was cooling and the dense cool layer could absorb the soft X-rays. This layer was not completely ionized like the outer circumstellar medium because of its high density.

The X-ray emission from supernovae is the primary radiation. It is responsible for heating and ionizing gas that radiates at ultraviolet/optical/infrared wavelengths. In the case of SN 1993J, emission lines from both the cool gas downstream from the radiative shock and from the freely expanding ejecta have been observed (Fransson et al., in preparation). By following the time dependence of the $\mathrm{X}$-ray emission together with the multiwavelength emission (radio through ultraviolet), it should be possible to construct the density profile of the exploded star.

SN 1993J is an example of the explosion of a red supergiant progenitor star into its dense surrounding wind. The resulting high X-ray luminosity (initially $\gtrsim 5 \times 10^{40} \mathrm{erg} \mathrm{s}^{-1}$ ) can be contrasted with the X-ray luminosity of SN 1987A ( $\lesssim 10^{35} \mathrm{erg} \mathrm{s}^{-1}$ ); the initial luminosity was even lower, due to the blue supergiant progenitor and its very low circumstellar density (Hasinger, Aschenbach, \& Trümper 1996). The X-ray luminosity has been rising since 1990, presumably because the supernova is interacting with gas in the red supergiant's wind, photoionized by the progenitor star, that extends in from the bright optical ring. The interaction of the forward shock front with the dense ring has started in one clump (Sonneborn et al. 1998). Over the next decade, when the shock front interacts with the dense red supergiant gas, the $\mathrm{X}$-ray luminosity will rise to $\gtrsim 10^{36} \mathrm{erg} \mathrm{s}^{-1}$ (Borkowski, Blondin, \& McCray 1997). As in the case of SN $1993 \mathrm{~J}$, this X-ray source will illuminate the ejecta of SN 1987A and other dense gases in the region.

\subsection{Supernova Remnants: Thermal Emission}

The X-ray study of supernova remnants has a long history and a large literature. Here, I will briefly comment on current directions in modeling the thermal emission from remnants.

One trend in the analysis of X-ray spectra is to model the spectrum in the absence of a specific hydrodynamic model for the remnant. Early X-ray observations were modeled by two-component ionization equilibrium. However, the ionization/recombination timescales are less than the age of the younger remnants, so nonequilibrium models are required. The conditions in an element of gas can then be described by the temperature and "ionization time," or $n_{e} t$, where $t$ is the time since being shocked and $n_{e}$ is the electron density. This method is especially effective when high-resolution spectra are available, as in the case of the high surface-brightness region of Puppis A (Canizares \& Winkler 
1981). However, even with the new generation of X-ray observatories, highresolution spectroscopy will be possible for only a limited number of objects.

Although plasma diagnostics can provide valuable information on a remnant, hydrodynamic models together with ionization calculations are necessary to obtain a more complete description of a remnant. In addition, multiwavelength observations are needed to provide a picture of the interaction situation. For example, the bright X-ray and radio ring in Cas A is frequently cited as emission from the reverse shock wave (e.g., Fabian et al. 1980). However, the optical, quasistationary flocculi suggest that this is the position of a slow, dense shell created by progenitor mass loss (Chevalier \& Liang 1989). A hydrodynamic model and emission calculation based on this scenario gives an estimated X-ray emitting mass of ejecta of $\sim 2 M_{\odot}$ (Borkowski et al. 1996), about a factor of 2 lower than previous estimates.

The complex surroundings of Cas A bear some relation to those observed around SN 1987A. Mass-loss phenomena probably play a role in another young remnant, Kepler (Borkowski, Blondin, \& Sarazin 1992). In general, core-collapse supernovae with massive star progenitors are expected to expand into complex surroundings created by mass loss.

In the case of Type Ia supernovae, which probably have white dwarf progenitors, interaction with the interstellar medium is more likely. The observed expansion of both Tycho's remnant and SN 1006 are suggestive of interaction with a constant-density ISM because their expansion rates are close to the $R \propto t^{0.4}$ expected in the Sedov solution. However, X-ray spectral imaging of Tycho's remnant shows inhomogeneities, in particular, an Fe clump and a Si clump on the east side of the remnant (Hwang \& Gotthelf 1997). Hughes (1996) finds that these clumps appear to be moving with an undecelerated velocity. In addition, the Fe ejecta appear to have different physical conditions from the ejecta of other elements (Hwang, Hughes, \& Gotthelf 1998). The implication is that the supernova ejecta are inhomogeneous. A plausible source of the inhomogeneity is the "Nickel bubble" effect (e.g., see Li, McCray, \& Sunyaev 1993 on SN 1987A).

The ejection of ${ }^{56} \mathrm{Ni}$ is expected for most supernovae, so inhomogeneous ejecta are likely to be present in most cases. This interaction has not yet been modeled in detail. The hydrodynamic evolution of remnants clearly cannot be calculated from first principles for any particular case. Modeling will have to take into account observations and plasma diagnostics for progress to be made.

\subsection{X-ray Synchrotron Emission}

The topic of X-ray synchrotron emission from supernova remnants was discussed in detail by Petre (2000, these Proceedings), and I will only comment on the subject here. In an early discussion of the apparent synchrotron emission from SN 1006, Reynolds \& Chevalier (1981) suggested a model in which particles are accelerated to high energy in a power-law energy spectrum at the shock front and experience synchrotron losses in the downstream region. The steady injection with synchrotron losses steepens the flux spectral index by 0.5 above the point where synchrotron losses become important. Sari, Piran, \& Narayan (1998) suggested a similar model for $\gamma$-ray burst afterglows. However, Reynolds (1996) found that the ASCA data (Koyama et al. 1995) could be better fitted by a model in which the shock acceleration of particles is limited by synchrotron 
emission, which causes an approximately exponential turnover of the spectrum. Under these conditions, the maximum frequency of the synchrotron emission depends primarily on the shock velocity, so it is expected that the relativistic shock generated by a $\gamma$-ray burst would be able to produce emission at high energy. The same should be true of the shock waves in extragalactic supernovae. However, downstream synchrotron losses appear to drop the nonthermal emission below the thermal emission in the cases where X-ray emission has been observed from a supernova in its early years.

\subsection{Gamma-ray Emission}

In the case of SN 1006, the high-energy electrons apparently give rise to observable TeV $\gamma$-ray emission, probably as a result of inverse Compton scattering with $2.7 \mathrm{~K}$ blackbody photons (Tanimori et al. 1998). This mechanism is probably not the cause of the lower energy $(\sim 100 \mathrm{MeV}-1 \mathrm{GeV}) \gamma$-ray emission that has been detected from supernova remnants by the EGRET experiment on CGRO (Esposito et al. 1996). The identifications cannot be considered completely secure, but the association with remnants that appear to be interacting with dense gas (e.g., IC 443, W44) gives some confidence in the associations. There have been a number of models of the high-energy emission from IC 443 and W44 including the processes of nonthermal bremsstrahlung, inverse Compton, and pion decay emission. Generally, these studies have concentrated on the emission processes and on the particle acceleration mechanism in the shock front but have assumed that the shock wave expansion is adiabatic (de Jager \& Mastichiadis 1997; Sturner et al. 1997; Gaisser, Protheroe, \& Stanev 1998; Baring et al. 1999). In fact, there is good evidence that the shock fronts in IC 443 and W44 are primarily in the radiative phase of evolution. In Chevalier (1999), I argued that these remnants are interacting with molecular clouds and that the shock fronts become radiative as they expand in the interclump medium of the cloud. Ambient cosmic rays are compressed and swept into the dense radiative shell where they can emit the $\gamma$-ray radiation. This process was examined in more detail by Bykov et al. (2000), who found that this particular scenario would only work if the energy spectrum of cosmic-ray electrons extends to low values. An alternative is that there is shock injection of electrons into the acceleration process. Bykov et al. (2000) found that this is a possibility, although it would require a substantial fraction of the shock energy going into the accelerated electrons.

The correlation between $\gamma$-ray emission from a remnant and evidence for molecular cloud interaction (Esposito et al. 1996) is generally expected because the high-energy emission should be stronger where interaction with a dense medium is taking place. The molecular cloud structure is expected to have dense, molecular clumps embedded within an interclump medium. In the model of Chevalier (1999) and Bykov et al. (2000), most of the $\gamma$-ray emission is produced in the dense, radiative shell in the interclump gas. This prediction can eventually be tested with GLAST. The shock waves in the dense clumps are sites of molecular line emission and are also potential sources of high-energy emission. Bykov et al. (2000) found that the slow shocks in the dense gas were unlikely to accelerate particles to energies where they would be significant sources of $\gamma$-ray bremsstrahlung emission or radio synchrotron emission. However, they could be sources of hard X-ray bremsstrahlung emission, although there is some 
uncertainty in whether the ionization necessary for the acceleration process is present. In ASCA observations, Keohane et al. (1997) did find a correlation of hard X-ray emission regions with regions of molecular emission. The Chandra Observatory will allow a much more detailed examination of this question.

\section{Radioactivity}

The study of high-energy emission from supernova remnants due to radioactivity started with observations of SN 1987A. The luminosity of SN 1987A implied that $0.07 M_{\odot}$ of ${ }^{56} \mathrm{Ni}$ was initially ejected (McCray 1993). The presence of ${ }^{56} \mathrm{Co}$ in SN 1987A was confirmed directly by the detection of ${ }^{56} \mathrm{Co} \gamma$-ray lines with balloon and satellite experiments (e.g., McCray 1993). The $\gamma$-ray lines, as well as continuum $\mathrm{X}$-rays from downscattered $\gamma$-rays, emerged more rapidly than had been predicted in one-dimensional models. This can be attributed to RayleighTaylor instabilities that occur when inner, dense layers are slowed down by outer, lower-density layers. The other radioactive species to be expected have increasing half-lives. After ${ }^{56} \mathrm{Co},{ }^{57} \mathrm{Co}$, with a half-life of 272 days, is expected to become the dominant power source. Direct evidence for the isotope came with the detection of the $\gamma$-ray line with the Compton Gamma-Ray Observatory (Kurfess et al. 1992). A mass of $0.003 M_{\odot}$ of ${ }^{57} \mathrm{Co}$ in SN 1987A was deduced to be present, which implies that the initial ${ }^{57} \mathrm{Ni} /{ }^{56} \mathrm{Ni}$ ratio in SN $1987 \mathrm{~A}$ was about 1.5 times the solar ratio of ${ }^{57} \mathrm{Fe} /{ }^{56} \mathrm{Fe}$.

The isotope ${ }^{57}$ Co became the dominant power source in SN 1987A at an age of about 1200 days but was overtaken by ${ }^{44} \mathrm{Ti}$ at an age of 1500 days. A mass of ${ }^{44} \mathrm{Ti}$ of $(1-2) \times 10^{-4} M_{\odot}$ has been deduced both on the basis of the light curve of SN 1987A and from analysis of the late optical spectrum (Chugai et al. 1997). ${ }^{44} \mathrm{Ti}$ has a half-life of 60 years (Ahmad et al. 1998) and can be expected to dominate the power to the inner ejecta nebula for future decades. At a distance of $50 \mathrm{kpc}$, the ${ }^{44} \mathrm{Ti} \gamma$-ray line from SN $1987 \mathrm{~A}$ is not accessible with current experiments. Even the upcoming INTEGRAL observatory is not assured of detecting the $\gamma$-ray line from SN 1987A. However, the ${ }^{44} \mathrm{Ti}$ line is accessible from nearby, young Galactic supernova remnants. It has been observed from Cas A (Iyudin et al. 1994) with an age of about 300 years and a distance of about $3 \mathrm{kpc}$, and from a previously undetected supernova superposed on the Vela supernova remnant (Iyudin et al. 1998). In both cases, the inferred mass is $(1-2) \times 10^{-4} M_{\odot}$, as in SN 1987A. This amount is at the high end of expectations for explosive nucleosynthesis in a massive star (Woosley \& Weaver 1995; Thielemann, Nomoto, \& Hashimoto 1996).

The field of $\gamma$-ray line observations is in its infancy. Most supernovae are thought to produce ${ }^{56} \mathrm{Ni}$ and its decay product, ${ }^{56} \mathrm{Co}$, but a sensitivity level at least an order of magnitude better than that expected for the INTEGRAL observatory will be necessary to detect supernovae on a regular basis.

\section{Conclusions and Future Prospects}

The launch of the Chandra Observatory and the expected launch of XMM and Astro-E should revolutionize the X-ray study of supernovae and their remnants. The complexity of the hydrodynamic interaction of supernovae with their sur- 
roundings has made it difficult to obtain reliable estimates of the abundances in young remnants. The improvements in spatial and spectral resolution should allow progress in this area and make possible a better understanding of the nature of supernova explosions and their progenitors. These missions should also help to clarify the relation between the thermal and the nonthermal X-ray emission from remnants. The nonthermal emission must be accounted for before a reliable interpretation of the thermal radiation can be carried out.

At higher energies ( $\gamma$-rays), the study of supernovae and their remnants is still in its infancy. The GLAST mission is needed to confirm the high-energy emission from remnants and to show its relation to molecular cloud interaction. The INTEGRAL observatory should give some increase in the number of $\gamma$-ray line sources, but a higher sensitivity mission is needed for this field to realize its full potential.

Acknowledgments. This work was supported in part by NASA grant NAG5-8232.

\section{References}

Ahmad, I., Bonino, G., Castagnoli, G. C., Fischer, S. M., Kutschera, W., \& Paul, M. 1998, Phys. Rev. Lett., 80, 2550

Baring, M. G., Ellison, D. C., Reynolds, S. P., Grenier, I. A., \& Goret, P. 1999, ApJ, 513, 311

Borkowski, K., Blondin, J. M., \& McCray, R. A. 1997, ApJ, 477, 821

Borkowski, K., Blondin, J. M., \& Sarazin, C. L. 1992, ApJ, 400, 222

Borkowski, K., Szymkowiak, A. E., Blondin, J. M., \& Sarazin, C. L. 1996, ApJ, 466,866

Bykov, A. M., Chevalier, R. A., Ellison, D. C., \& Uvarov, Y. A. 2000, ApJ, submitted

Canizares, C. R., \& Winkler, P. F. 1981, ApJ, 246, L33

Chevalier, R. A. 1998, Mem. Soc. Astr. Italy, 69, 977

Chevalier, R. A. 1999, ApJ, 511, 798

Chevalier, R. A., \& Liang, E. P. 1989, ApJ, 344, 332

Chugai, N. N., Chevalier, R. A., Kirshner, R. P., \& Challis, P. M. 1997, ApJ, 483,925

de Jager, O. C., \& Mastichiadis, A. 1997, ApJ, 482, 874

Ensman, L., \& Burrows, A. 1992, ApJ, 393, 742

Esposito, J.A., Hunter, S. D., Kanbach, G., \& Sreekumar, P. 1996, ApJ, 461, 820

Fabian, A. C., Willingale, R., Pye, J. P., Murray, S. S., \& Fabbiano, G. 1980, MNRAS, 193, 175

Falk, S. W. 1978, ApJ, 225, L133

Fransson, C., Lundqvist, P., \& Chevalier, R. A. 1996, ApJ, 461, 993

Gaisser, T. K., Protheroe, R. J., \& Stanev, T. 1998, ApJ, 492, 219

Hasinger, G., Aschenbach, B., \& Trümper, J. 1996, A\&A, 312, L9 
Hughes, J. P. 1996, BAAS, 28, 1334

Hwang, U., \& Gotthelf, E. V. 1997, ApJ, 475, 665

Hwang, U., Hughes, J. P., \& Petre, R. 1998, ApJ, 497, 833

Iyudin, A. F., et al. 1994, A\&A, 284, L1

Iyudin, A. F., et al. 1997, Nature, 396, 142

Keohane, J. W., Petre, R., Gotthelf, E. V., Ozaki, M., \& Koyama, K. 1997, ApJ, 484, 350

Kirshner, R. P., Sonneborn, G., Crenshaw, D. M., \& Nassiopoulos, G. E. 1987, ApJ, 320, 602

Klein, R. I., \& Chevalier, R. A. 1978, ApJ, 223, L109

Koyama K., Petre R., Gotthelf, E. V., Hwang, U., Matsuura, M., Ozaki, M., \& Holt, S. S. 1995, Nature, 378, 255

Kurfess, J. D., et al. 1992, ApJ, 399, L137

Li, H., McCray, R., \& Sunyaev, R. A. 1993, ApJ, 419, 824

Lundqvist, P., \& Fransson, C. 1996, ApJ, 464, 924

Matzner, C. D., \& McKee, C. F. 1999, ApJ, 510, 379

McCray, R. 1993, ARA\&A, 31, 175

Reynolds, S. P. 1996, ApJ, 459, L13

Reynolds, S. P., \& Chevalier, R. A. 1981, ApJ, 245, 912

Sari, R., Piran, T., \& Narayan, R. 1998, ApJ, 497, L17

Sonneborn, G., et al. 1998, ApJ, 492, L139

Sturner, S. J., Skibo, J. G., Dermer, C. D., \& Mattox, J. R. 1997, ApJ, 490, 619

Tanimori, T., et al. 1998, ApJ, 497, L25

Thielemann, F.-K., Nomoto, K., \& Hashimoto, M. 1996, ApJ, 460, 408

Woosley, S. E., \& Weaver, T. A. 1995, ApJS, 101, 181 